\title{
ERRATUM
}

\section{Bacillus gaemokensis sp. nov., Isolated from Foreshore Tidal Flat Sediment from the Yellow Sea}

\author{
Min-Young Jung ${ }^{1,5}$, Woon Kee Paek ${ }^{2}$, In-Soon Park ${ }^{1}$, Jeong-Ran Han ${ }^{1}$, Yeseul Sin ${ }^{1}$, \\ Jayoung Paek ${ }^{1}$, Moon-Soo Rhee ${ }^{1}$, Hongik Kim ${ }^{3}$, Hong Seok Song ${ }^{4}$, and Young-Hyo Chang ${ }^{1 *}$ \\ ${ }^{1}$ Korean Collection for Type Cultures, Biological Resource Center, KRIBB, Daejeon 305-806, Republic of Korea \\ ${ }_{2}^{2}$ National Science Museum, Daejeon 305-705, Republic of Korea \\ R\&D Division, Vitabio Inc., Daejeon 300-824, Republic of Korea \\ ${ }^{4} R \& D$ Division, Korea Gas Corporation, Ansan 426-790, Republic of Korea \\ ${ }^{5}$ Department of Microbiology, College of Medicine, Chung-Ang University, Seoul 156-756, Republic of Korea \\ (Received April 16, 2010 / Accepted August 16, 2010)
}

In the article by Jung et al. that appears in the Journal of Microbiology 2010; 48, 876-871. Page 867 first author name Min-Young Jung should be changed as Min Young Jung. 